\title{
Microscope Integrated Intraoperative Optical Coherence Tomography-Guided DMEK in Corneas with Poor Visualization
}

This article was published in the following Dove Press journal: Clinical Ophthalmology

\section{Namrata Sharma $(\mathbb{D}$ \\ Pranita Sahay \\ Prafulla K Maharana \\ Praveen Kumar \\ Saima Ahsan \\ Jeewan S Titiyal}

Dr. Rajendra Prasad Centre for Ophthalmic Sciences, All India Institute of Medical Sciences, New Delhi, India

\section{Video Abstract}

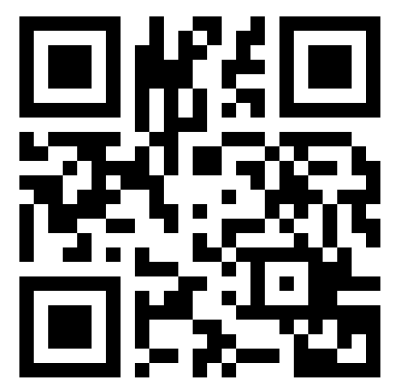

Point your SmartPhone at the code above. If you have a QR code reader the video abstract will appear. Or use: https://youtu.be/gvVe6EOEWJE
Correspondence: Namrata Sharma Department of Ophthalmology, Dr. Rajendra Prasad Centre for Ophthalmic

Sciences, All India Institute of Medical

Sciences, Room- 482, 4th Floor, New

Delhi I I0029, India

Tel +9l 9810856988

Email namrata.sharma@gmail.com
Purpose: To assess the utility of microscope-integrated intraoperative optical coherence tomography (Mi-OCT) for performing Descemet membrane endothelial keratoplasty (DMEK) in corneas with poor visualization.

Methods: It is a prospective interventional case series that included 25 consecutive cases of corneal decompensation with poor visualization that underwent Mi-OCT-guided DMEK at a tertiary eye care centre. The main outcome measures were graft attachment on day 3 and requirement for re-bubbling.

Results: The etiology for corneal decompensation was pseudophakic bullous keratopathy ( $\mathrm{n}=17)$, Fuchs endothelial corneal dystrophy $(n=4)$, failed graft $(n=2)$, iridocorneal endothelial syndrome $(n=1)$ and failed Descemet stripping automated endothelial keratoplasty $(n=1)$. Complete graft attachment was noted in $72 \%$ of cases. Graft detachment was noted in $16 \%$ of cases which required re-bubbling. No intervention was done for shallow peripheral detachment $(n=2)$ and peripheral Descemet membrane (DM) fold $(\mathrm{n}=1)$. All grafts were attached at six-months follow-up. The mean corrected distance visual acuity and central corneal thickness improved from $1.4 \pm 0.5 \operatorname{logMAR}$ and $799.6 \pm 110.9 \mu \mathrm{m}$ at baseline to $0.3 \pm 0.3 \operatorname{logMAR}$ and $536.28 \pm 11.44 \mathrm{um}$ at six months. Mi-OCT was helpful in visualizing areas of peripheral anterior synechiae, missing DM, retained DM tags after descemetorhexis, DMEK roll configuration and orientation in the injector and anterior chamber, interface fluid and peripheral folds in the DMEK graft.

Conclusion: Mi-OCT helps in identification of the anatomy and dynamics of the host DM, DM roll and anterior chamber in cases with poor visualization and is a useful tool while performing DMEK in such cases.

Keywords: DMEK, descemet membrane endothelial keratoplasty, iOCT, Mi-OCT, microscope-integrated intra-operative optical coherence tomography

\section{Introduction}

DMEK results in faster visual rehabilitation with better visual outcome and reduced risk of graft rejection. ${ }^{1,2}$ However, there are several challenges in the surgical technique like graft preparation, unfolding of the DMEK graft in the anterior chamber, difficulty in visualizing the DMEK roll in hazy cornea and postoperative risk of detachment. ${ }^{3-9}$ These challenges have limited the use of this technique to cases with relatively clear cornea and normal anterior segment anatomy.

Unlike most of the published series in DMEK, the majority of our cases present at a delayed stage of corneal endothelial decompensation making them either unsuitable or surgically challenging for an endothelial keratoplasty. ${ }^{10}$ Several modifications have 
been suggested to overcome the difficulty of poor visualization. Burkhart et al described the use of intraoperative handheld slit lamp in these cases; however surgical sterility is at risk along with interruption of the surgical procedure with its use. ${ }^{11}$ Jacob et al described the intraoperative use of an endoilluminator to enhance the graft visualization based on reflexes from the edges of the graft in cases with poor corneal clarity. $^{12}$

Microscope integrated optical coherence tomography (Mi-OCT) gives a real-time cross-sectional image of the eye without interrupting the surgical procedure. This may be extremely useful while performing DMEK in the presence of advanced corneal decompensation or in cases of chronic corneal decompensation with corneal haze, which is often encountered in the majority of our cases. There are several studies on the role of Mi-OCT in DMEK; however very few studies have focused in its role in cases with hazy cornea. In the current study, we describe the role of MiOCT for better intraoperative visualization in cases with poor corneal clarity.

\section{Materials and Methods}

In this prospective interventional study, all cases of corneal decompensation with poor visualization presenting between July 2017 and June 2018 at a tertiary eye care centre were recruited after obtaining written informed consent. Inclusion criteria were cases with corneal decompensation and presence of severe corneal edema (CCT $>800 \mu \mathrm{m})$ or stromal haze due to chronic corneal decompensation that resulted in poor visualization of the anterior segment. (Supplementary Figure 1). Cases with central leucomatous corneal opacity obscuring the visual axis and significant iris anomaly were excluded. The study was approved by the institutional review board (Institute Ethics Committee, All India Institute of Medical Sciences, New Delhi, India; Ref no. IEC/NP-347/10.09.2015, RP-9/ Feb-2016) and adhered to the tenets of the Declaration of Helsinki. Confidentiality of patients' personal medical information was ensured. Preoperative demography, indication for surgery, uncorrected distance visual acuity (UCVA), corrected distance visual acuity (CDVA), intraocular pressure (IOP), anterior chamber details, central corneal thickness (CCT) on anterior segment coherence tomography (ASOCT), lens status and posterior segment details were recorded. All cases underwent Mi-OCT guided DMEK. Surgery was performed by a single surgeon (NS). The following points were noted intraoperatively-Descemet membrane (DM) tags after descemetorhexis, orientation of DM roll in the injector and anterior chamber, interface fluid after injection of air, presence of peripheral folds in the graft. Postoperatively, the cases were followed up on days 1, 3, and 7, 1 month, 3 months, and 6 months. The postoperative assessment included UCVA, CDVA, IOP, detailed slit lamp examination of the anterior segment, central corneal thickness (CCT) on anterior segment coherence tomography (ASOCT) and endothelial cell count on specular microscopy. The main outcome measures were graft attachment on day 3 and requirement for re-bubbling.

\section{Surgical Technique}

In this study, we used the microscope-integrated intraoperative optical coherence tomography (OPMI Lumera 700 and RESCAN 700, Carl Zeiss, Meditec, AG, Jena, Germany). This system has an optical coherence tomography (OCT) system integrated with the microscope and gives a live heads-up display to the surgeon on the right ocular of the microscope as well as an external video display. The surgeon can shift the point of focus of OCT with the foot pedal control in this system during surgery. An external assistant control of OCT is possible. RESCAN 700 works at a wavelength of 840 $\mathrm{nm}$ and captures 27,000 A-scans per second. It provides an axial resolution of $5 \mu \mathrm{m}$.

\section{Donor Cornea Preparation}

The donor cornea was placed over a Teflon block and stained with trypan blue dye for 30 seconds followed by partial thickness trephination with a $9 \mathrm{~mm}$ handheld disposable trephine (Nanoedge Ophthalmic Blades, Madhu Instruments, New Delhi, India). The peripheral rim of Descemet-endothelium complex was peeled off. Donor cornea was stained again with trypan blue dye for 30 seconds. The Descemet-endothelium complex was peeled with the help of McPherson forceps till $80 \%$ of the tissue was detached. A $3 \mathrm{~mm}$ trephine was then used to punch the stromal bed following which the Descemet endothelium complex was laid back on the stromal bed. The donor cornea was then inverted with the epithelium side up. The trephined tissue was lifted to expose the DM and an " $S$ stamp" (Moria, Antony, France) was used to mark the DM endothelium complex. ${ }^{13,14}$

Mi-OCT (RESCAN 700, Carl Zeiss Meditec) was used to check if there were any gaps between the Descemet endothelium complex and the underlying stromal bed prior to trephination. (Figure 1A and B) This step avoids irregular cuts during trephination. Final trephination varied from 7 to $8 \mathrm{~mm}$ based on the patient's corneal diameter. The DMEK roll was then loaded on a DMEK injector (Geuder AG, Heidelberg, Germany). The configuration of 


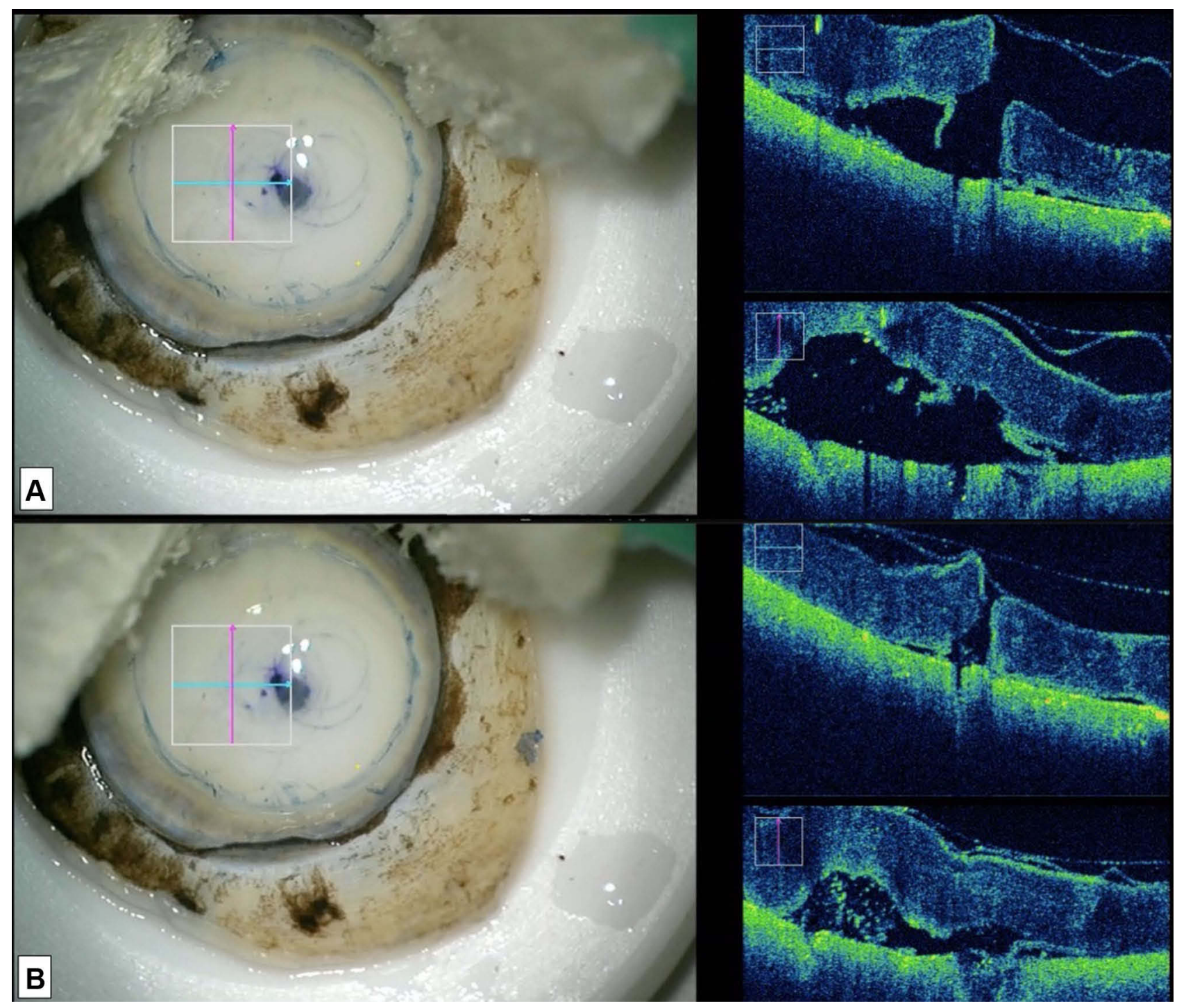

Figure I Intraoperative images of donor tissue preparation before trephining the peeled graft (A) showing fluid between the peeled DMEK graft and stromal bed on MiOCT; (B) showing the decrease in fluid space with the peeled DMEK graft falling back on the stromal bed after drying the edges with a Merocel wick on Mi-OCT.

DMEK roll was checked in the DMEK injector prior to injecting in the anterior chamber.

\section{Host Cornea Preparation}

The host preparation involved making a $3.2 \mathrm{~mm}$ clear corneal incision and two side ports. Descemetorhexis, inferior peripheral iridotomy and graft insertion using a Geuder injector was performed similar to the previously described technique for DMEK. ${ }^{5,15}$

Mi-OCT was used at various stages during this procedure. Before commencing the surgery, the posterior stroma/DM was assessed for presence of any focal abnormalities such as DM scar, prominent DM fold, and areas of missing DM. Also, areas of peripheral anterior synechiae as well as inner edge of the previous surgical scar were screened. This helped in better planning of the surgical site. After the descemetorhexis, Mi-OCT was used to screen the entire posterior corneal surface for any retained DM tags. (Figure 2A and B) Scoring and peeling were avoided in areas of missing Descemet membrane, delineated on Mi-OCT, in cases of complicated pseudophakic bullous keratopathy (PBK) to avoided undue damage to the corneal stroma.

\section{DMEK Roll in the Anterior Chamber}

The orientation of DMEK roll in the injector was checked with Mi-OCT prior to its injection in the anterior chamber (Figure 3A and B). The bevel was adjusted accordingly to ensure injection of the DMEK roll into the anterior chamber with the endothelial side 


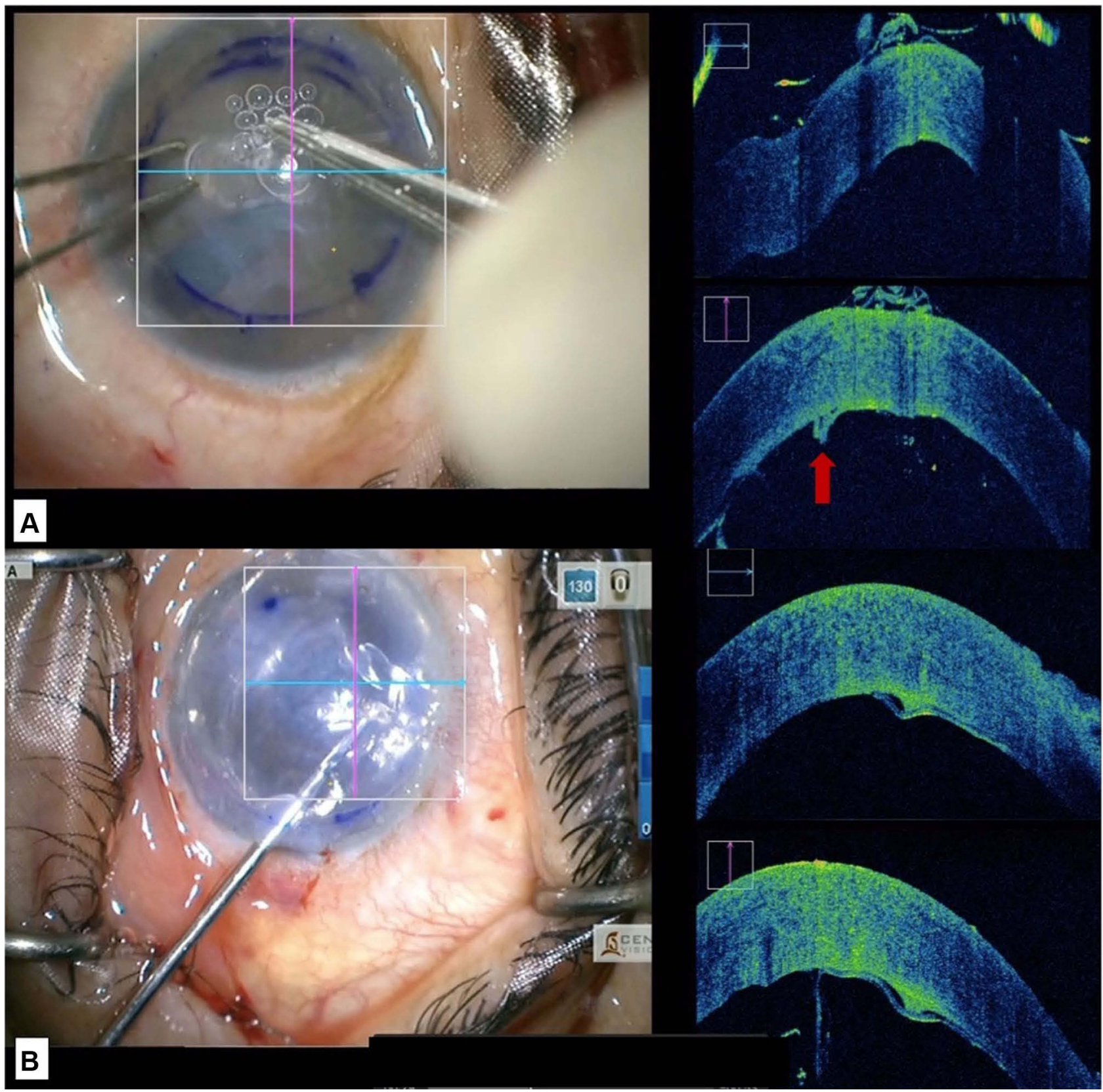

Figure 2 Intraoperative images (A) showing retained DM tag (marked with red arrow) after descemetorhexis; (B) removal of a retained DM tag with intravitreal forceps.

of DMEK roll-up so as to allow an easy unfolding of the graft in the anterior chamber in the correct orientation. After injection, prior to unfolding the DMEK roll in the anterior chamber, its configuration was again confirmed on Mi-OCT. In cases of inappropriate orientation, the DMEK roll was flipped with the help of balanced salt solution (BSS) followed by careful injection of air beneath the graft using a 27-gauge cannula. This helps in unfolding the graft in the correct orientation with minimal chance of obtaining an "inverted S"
(Figure 4A-C). After unfolding the DMEK graft, the "S mark" was checked to confirm the appropriate orientation of the graft (Figure 4D). The edge of the graft was then assessed for the presence of peripheral folds. In cases where the graft was well attached (without any interface fluid) with a peripheral fold, wherein the endothelium was rolled up, BSS was injected into the interface (between the host stroma and the graft) at the site of the fold with a 27-gauge cannula to induce detachment and unfolding of the graft. This was again 


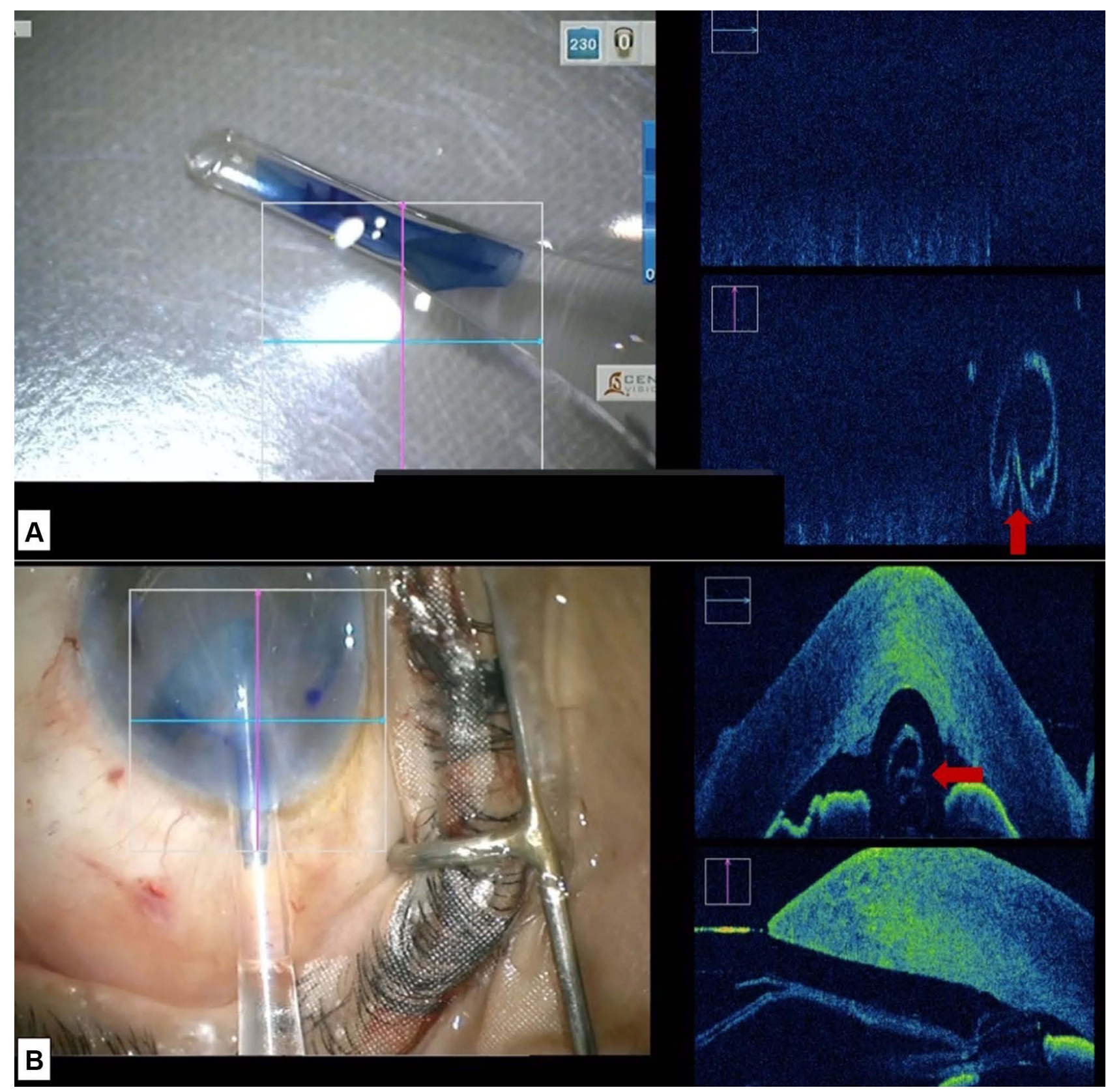

Figure 3 Intraoperative image showing DMEK roll orientation in the DMEK injector (A) prior to injection (the graft is inverted with DM facing down as highlighted by the red arrow); (B) at the time of injection (the graft is oriented with the DM towards the right as highlighted by the red arrow).

followed by air injection into the anterior chamber. In cases with a peripheral fold where the graft was well attached (without any interface fluid), wherein the Descemet was rolled down, BSS was injected below the graft, in the area of the fold to induce unfolding. This was followed by air injection into the anterior chamber (Figure 5A-C). At the end of the procedure, Mi-OCT was used to look for interface fluid, that was visible as a gap or dark band between the host and donor tissue. Corneal surface was then gently massaged with a 27 gauge-cannula until these interface gaps were eliminated.

\section{Results}

\section{Baseline Characteristics}

A total of 25 cases underwent Mi-OCT guided DMEK in corneas with poor visualization. The mean age of cases was 57.6 years (range: $41-70$ years) with 11 males and 14 females. (Supplementary Table 1). The etiology was pseudophakic corneal edema in 17 cases, Fuchs endothelial corneal 


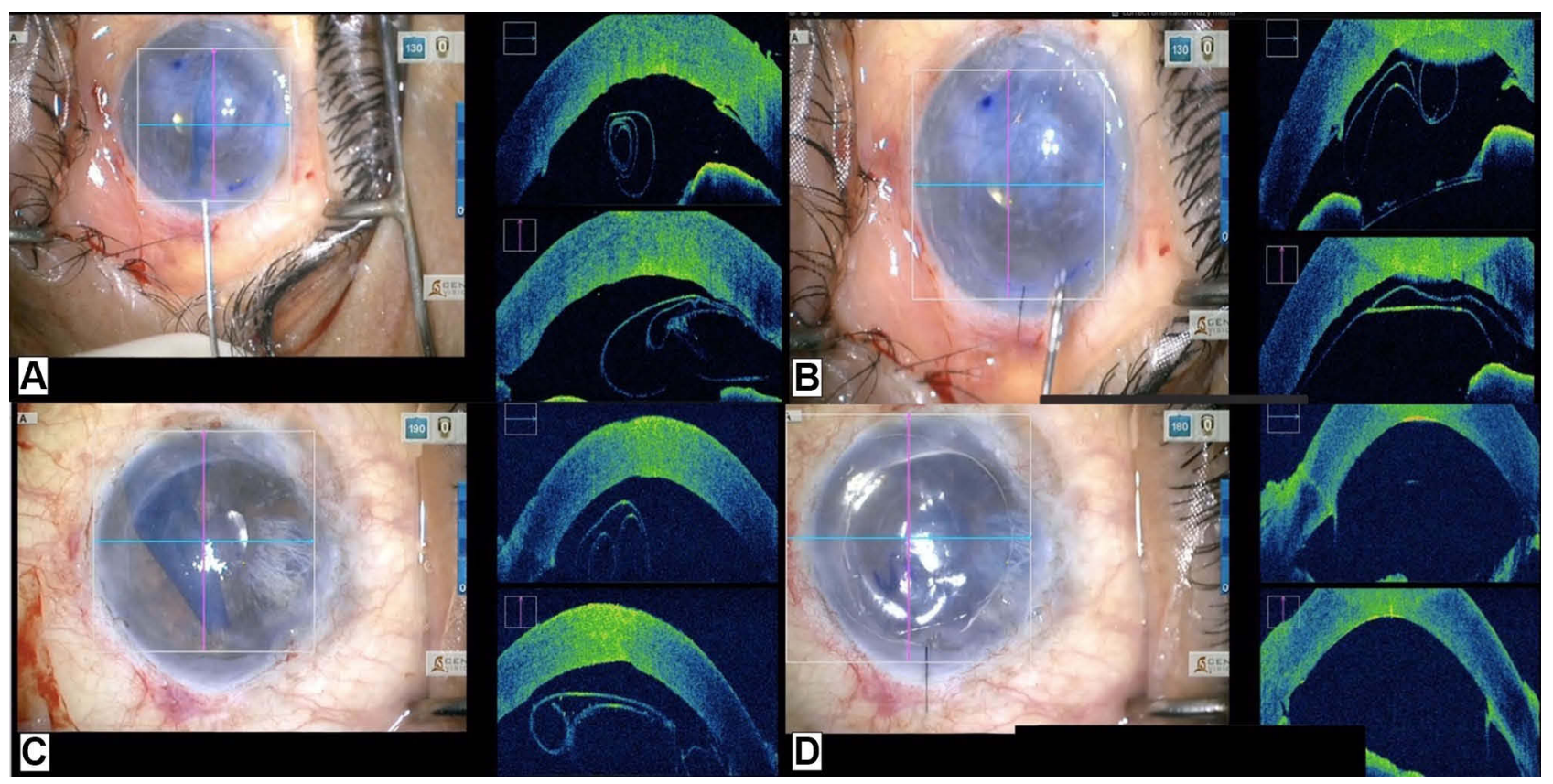

Figure 4 Intraoperative images showing (A) tight scroll in anterior chamber; (B) double scroll with Descemet up and endothelium down (correct orientation); (C) double scroll with Descemet down and endothelium up (incorrect orientation); (D) well attached DMEK graft in a case of failed graft.

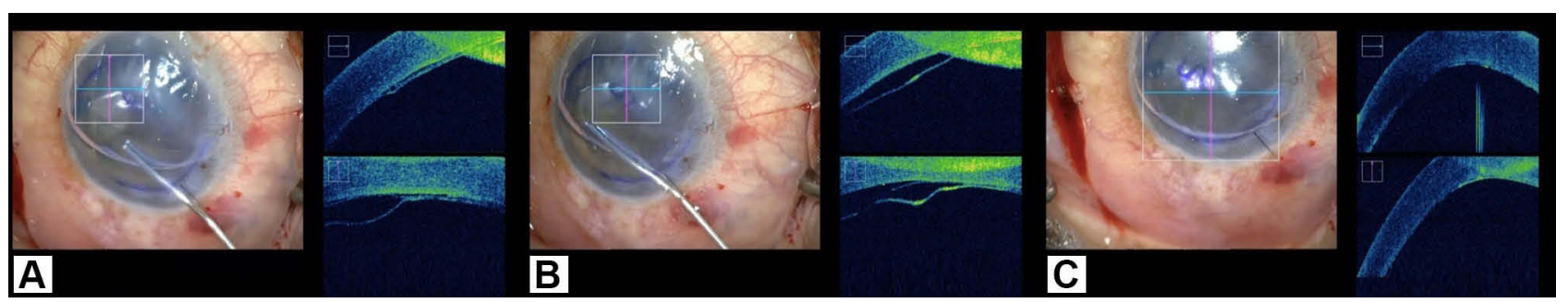

Figure 5 Intraoperative image showing (A) Fold at the edge of the graft with the DM folded down after injection of air; (B) injection of BSS below the graft at the site of fold to induce unfolding; (C) completely unfolded and attached graft.

dystrophy (FECD) in four cases, failed graft in two cases, iridocorneal endothelial (ICE) syndrome and failed Descemet stripping automated endothelial keratoplasty (DSAEK) in one case each. The baseline mean CDVA was $1.4 \pm 0.5 \log$ MAR units (range: $0.78-2 \log$ MAR). The baseline mean CCT was $799.6 \pm 110.9 \mu \mathrm{m}$ (range: $620-980 \mu \mathrm{m}$ ). The mean IOP at baseline was $12 \pm 3.4 \mathrm{mmHg}$.

\section{Intraoperative Findings}

In two cases the location of $3.2 \mathrm{~mm}$ surgical incision was modified to avoid the scar due to previous surgery which was detected on Mi-OCT. Areas of missing DM, due to previous surgical trauma were visualized well in $12 \%$ of the cases $(n=3 / 25)$. This guided the surgeon to avoid undue scrapping and damage to stroma in the areas of absent DM. During host cornea descemetorhexis, retained DM tags were noted in Mi-OCT in $92 \%$ of cases $(n=23 / 25)$ which were otherwise not visible. In all these cases, the DM tag could be removed under Mi-OCT guidance using micro-vitreoretinal forceps and vitrectomy cutter. In case of ICE syndrome $(n=1)$, Mi-OCT guided assessment of peripheral and mid-peripheral anterior synechiae and its release was done prior to graft injection.

The orientation and shape of the DM roll in the cartridge could be identified in all cases with Mi-OCT. Most of the cases had a double scroll $(n=19 / 25)$, while four cases had a tight scroll (16\%) and two cases had a trifold $(8 \%)$. After insertion of the graft the orientation of the graft could be correctly identified in all cases. In 18 cases the graft orientation on Mi-OCT correlated with the $\mathrm{S}$ mark orientation. In seven cases $(28 \%)$ the $\mathrm{S}$ mark was not visible due to the presence of hazy media; however the orientation could be assessed on Mi-OCT in all these cases (correct orientation three; incorrect orientation four). 
In three cases, wherein the graft appeared to be attached on microscope view following air injection, interface fluid was detected on Mi-OCT which cleared with over-pressurization of the globe with air injection. A peripheral fold of the DMEK graft was noted on Mi-OCT in three cases (upward fold two; downward fold one) with successful unfolding in two of the three cases. Successful attachment of the graft was noted at the end of surgery in all cases.

\section{Postoperative Outcomes}

Complete attachment of the graft was noted in $72 \%(n=18)$ cases on day 3. Shallow peripheral detachment was noted in two cases and peripheral Descemet membrane fold was noted in one case which did not require any surgical intervention. In $16 \%(n=4)$ of the cases graft detachment was noted which required rebubbling. At six months follow-up all the grafts were attached. The mean CDVA and CCT at six months follow-up was $0.3 \pm 0.3 \operatorname{logMAR}$ and $536.2 \pm 11.4 \mu \mathrm{m}$. Postoperative raised IOP $(>21 \mathrm{mmHg})$ was noted in two cases that was managed with topical anti-glaucoma medication. All cases had clear cornea at six months follow-up except for three cases that had residual stromal haze. The endothelial cell loss was 36\% (range: 26-46\%) at six months follow-up.

\section{Discussion}

Performing DMEK in eye with corneal haze is often difficult. Mi-OCT was useful in several steps while performing DMEK in our series that included cases undergoing DMEK in presence of corneal haze. Identification of posterior stromal irregularities and scarring due to previous surgery helped us in modifying the location of our main incision to avoid problem of leaky wound due to poor wound apposition. Areas of incomplete descemetorhexis could be picked up which allowed complete removal of the residual DM tags. Identification of areas of missing DM in the host cornea prior to descemetorhexis prevented the unnecessary attempts of DM scrapping which can otherwise result in postoperative stromal haze. Detection of shallow areas of detachment that could have been missed in otherwise hazy corneas could be picked up with the help of Mi-OCT. Precise location and extent of peripheral DM folds allowed us to decide whether to attempt its unfolding or not. This reduced the inadvertent damage to the graft with repeated attempts of unfolding the graft. The use of S mark or four asymmetrical marks have been described to identify correct orientation of the DM roll prior to air injection; however, in most of our cases, the exact orientation of $\mathrm{S}$ mark was difficult to identify due to corneal haze. ${ }^{13,14,16}$ In some cases, although the $\mathrm{S}$ mark was apparent in the beginning of the surgery, it became difficult to identify it with passage of time. In all these cases, Mi-OCT helped us in identifying the orientation of the DM roll and correct localization of the endothelial side. The usefulness of MiOCT was especially appreciated in seven cases wherein the media was extremely hazy precluding the assessment of the graft as well as the anterior segment. An inverted graft orientation, which could have been otherwise missed, was recognised in $12 \%(n=3 / 25)$ cases which was flipped with BSS prior to air injection. Lastly, although not that important, it allowed us to be confident about the complete air fill of the anterior chamber with precise attachment of the graft.

Steven et al in 2013 first reported the use of Mi-OCT (iOCT; optomedical Technologies, Gmbh) in cases undergoing DMEK. ${ }^{17}$ In this retrospective study, the authors highlighted several advantages of Mi-OCT such as monitoring of graft rolling behaviour, precise identification of the endothelial side of the graft, visualizing of DM tags of donor cornea, and DM apposition to the stroma.

In a subsequent study, a part of the multicentric DISCOVER study, Cost et al reported the experience of a novice DMEK surgeon in eight cases undergoing Mi-OCT guided DMEK (Rescan 700, Carl Zeiss Meditec) ${ }^{18}$ The authors reported that during surgery Mi-OCT was useful in identification of the DM tags, graft orientation, and graft attachment. Similar findings were noted by Saad et al in a prospective study including 14 cases that underwent Mi-OCT guided DMEK (Rescan 700, Carl Zeiss Meditec). ${ }^{19}$

Most of these studies included cases where the corneal clarity was relatively better and Mi-OCT was used as a supplemental tool. The results of our study are similar to the above study. It is important to note that we achieved similar results despite of increased level of difficulty in our cases, in contrast to the above-mentioned studies.

Various modifications have been proposed to overcome the difficulty of poor visualization during DMEK procedure. These modifications include chandelier illumination by Shimizu et al, transcorneal illumination by Jacob et al and Kobayashi et al. ${ }^{12,20,21}$ Use of chandelier illumination has several limitations such as need for a dilated pupil that could cause problems in graft unfolding, increased risk of endothelial damage. ${ }^{21}$ Endoilluminator-assisted DMEK allows easy identification of graft orientation as well as easy visualization of the graft during all steps of surgery. ${ }^{12}$ However, the need for an assistant, and crowding of surgical space are of concern. Moreover, both of these techniques cannot provide the detailed cross-section view of 
cornea and detection of retained DM tags, interface fluid and deeper corneal scar could not be done with this technique. In contrast, Mi-OCT, in addition to the benefits of the above-mentioned techniques it provides a real-time cross-sectional monitoring of the anterior chamber, cornea and the DM roll and their interaction throughout the DMEK procedure.

To conclude, hazy cornea is considered a relative contraindication while selecting a case for DMEK as it results in poor visualizing of the DM tags, DMEK roll, and S mark. Mi-OCT is an extremely useful tool in this situation. Just to highlight, DMEK could be performed successfully in all our cases including difficult cases like ICE syndrome. A huge number of cases which are otherwise rendered unsuitable for DMEK, due to the presence of severe corneal edema and poor visibility of the anterior segment details, can be safely taken up for this surgery in the presence of Mi-OCT.

The lack of control arm and small sample size are the limitations of the current study. However, it is not practically possible to conduct a randomized controlled trial in such a situation and this number of samples is fairly adequate to draw evidence in favour of the device. The cost and availability of Mi-OCT is a concern. Thus, we do not recommend this in routine cases of DMEK. However, Mi-OCT should be used while doing DMEK in corneas with significant corneal haze. In an experienced hand MiOCT may not add any extra benefit but for a novice surgeon in DMEK and cases with poor visibility it is an extremely useful tool.

\section{Conclusion}

Mi-OCT is a useful tool for performing DMEK. It can guide the surgeon in various surgical steps, especially in difficult cases with poor visualization.

\section{Abbreviations}

Mi-OCT, microscope-integrated optical coherence tomography; DMEK, Descemet membrane endothelial keratoplasty; EK, endothelial keratoplasty; PLK, posterior lamellar keratoplasty; DM, Descemet membrane; UCVA, uncorrected visual acuity; CDVA, corrected distance visual acuity; IOP, intraocular pressure; CCT, central corneal thickness; ASOCT, anterior segment optical coherence tomography; OCT, optical coherence tomography; PBK, pseudophakic bullous keratopathy; FECD, Fuch's endothelial corneal dystrophy; ICE, iridocorneal endothelial syndrome; DSAEK, Descemet stripping automated endothelial keratoplasty.

\section{Disclosure}

The authors report no conflicts of interest in this work.

\section{References}

1. Price MO, Gupta P, Lass J, Price FW Jr. EK (DLEK, DSEK, DMEK): new Frontier in cornea surgery. Annu Rev Vis Sci. 2017;3:69-90. doi:10.1146/annurev-vision-102016-061400

2. Singh A, Zarei-Ghanavati M, Avadhanam V, Liu C. Systematic review and meta-analysis of clinical outcomes of descemet membrane endothelial keratoplasty versus descemet stripping endothelial kerato - PubMed - NCBI. Available from: https://www.ncbi.nlm.nih. gov/pubmed/28834814. Accessed July 23, 2018.

3. Melles GRJ, Ong TS, Ververs B, van der Wees J. Descemet membrane endothelial keratoplasty (DMEK). Cornea. 2006;25(8):987990. doi:10.1097/01.ico.0000248385.16896.34

4. Maharana PK, Sahay P, Titiyal JS, Sharma N. Sinsky hook assisted roll preparation (SHARP): a modified technique for Descemet membrane endothelial keratoplasty donor preparation. Saudi J Ophthalmol. 2018. doi:10.1016/j.sjopt.2018.12.002

5. Terry MA, Straiko MD, Veldman PB, et al. Standardized DMEK technique: reducing complications using prestripped tissue, novel glass injector, and Sulfur Hexafluoride (SF6) gas. Cornea. 2015;34 (8):845-852. doi:10.1097/ICO.0000000000000479

6. Siebelmann S, Gehlsen U, Le Blanc C, Stanzel TP, Cursiefen C, Steven P. Detection of graft detachments immediately following Descemet membrane endothelial keratoplasty (DMEK) comparing time domain and spectral domain OCT. Graefes Arch Clin Exp Ophthalmol. 2016;254(12):2431-2437. doi:10.1007/s00417-0163484-x

7. Gorovoy MS. DMEK complications. Cornea. 2014;33(1):101-104. doi:10.1097/ICO.0000000000000023

8. Muraine M, He Z, Toubeau D, et al. Validation of a novel device to easy the preparation of endothelial graft for DMEK. Acta Ophthalmol (Copenh). 2014;92. doi:10.1111/j.1755-3768.2014.4771.x

9. Yu CQ, Ta CN, Terry MA, Lin CC. Successful DMEK after intraoperative graft inversion. Cornea. 2015;34(1):97-98. doi:10.1097/ ICO.0000000000000311

10. Sharma N, Sachdev R, Pandey RM, et al. Study of factors for unsuitability of DSAEK in cases of corneal decompensation following cataract surgery. Int Ophthalmol. 2012;32(4):313-319. doi:10.1007/s10792-012-9521-9

11. Burkhart ZN, Feng MT, Price MO, Price FW. Handheld slit beam techniques to facilitate DMEK and DALK. Cornea. 2013;32(5):722724. doi:10.1097/ICO.0b013e31827797e7

12. Jacob S, Agarwal A, Agarwal A, Narasimhan S, Kumar DA, Sivagnanam S. Endoilluminator-assisted transcorneal illumination for Descemet membrane endothelial keratoplasty: enhanced intraoperative visualization of the graft in corneal decompensation secondary to pseudophakic bullous keratopathy. J Cataract Refract Surg. 2014;40(8):1332-1336. doi:10. 1016/j.jcrs.2014.06.019

13. Veldman PB, Dye PK, Holiman JD, et al. The S-stamp in descemet membrane endothelial keratoplasty safely eliminates upside-down graft implantation. Ophthalmology. 2016;123(1):161-164. doi:10.1016/j. ophtha.2015.08.044

14. Bachmann BO, Laaser K, Cursiefen C, Kruse FE. A method to confirm correct orientation of descemet membrane during descemet membrane endothelial keratoplasty. Am J Ophthalmol. 2010;149 (6):922-925.e2. doi:10.1016/j.ajo.2010.01.005

15. Hamzaoglu EC, Straiko MD, Mayko ZM, Sáles CS, Terry MA, First T. 100 eyes of standardized descemet stripping automated endothelial keratoplasty versus standardized descemet membrane endothelial keratoplasty. Ophthalmology. 2015;122(11):2193-2199. doi:10.1016/j. ophtha.2015.07.003 
16. Matsuzawa A, Hayashi T, Oyakawa I, et al. Use of four asymmetric marks to orient the donor graft during Descemet's membrane endothelial keratoplasty. BMJ Open Ophthalmol. 2017;1(1):e000080. doi:10.1136/ bmjophth-2017-000080

17. Steven P, Le Blanc C, Velten K, et al. Optimizing descemet membrane endothelial keratoplasty using intraoperative optical coherence tomography. JAMA Ophthalmol. 2013;131(9):1135-1142. doi:10.1001/ jamaophthalmol.2013.4672

18. Cost B, Goshe JM, Srivastava S, Ehlers JP. Intraoperative optical coherence tomography-assisted descemet membrane endothelial keratoplasty in the DISCOVER study. Am J Ophthalmol. 2015;160 (3):430-437. doi:10.1016/j.ajo.2015.05.020
19. Saad A, Guilbert E, Grise-Dulac A, Sabatier P, Gatinel D. Intraoperative OCT-assisted DMEK: 14 consecutive cases. Cornea. 2015;34(7):802-807. doi:10.1097/ICO.0000000000000462

20. Kobayashi A, Yokogawa H, Yamazaki N, Masaki T, Sugiyama K. The use of endoillumination probe-assisted Descemet membrane endothelial keratoplasty for bullous keratopathy secondary to argon laser iridotomy. Clin Ophthalmol. 2015;9:91-93. doi:10.2147/OPTH.S74981

21. Shimizu T, Hayashi T, Yuda K, et al. Chandelier illumination for descemet membrane endothelial keratoplasty. Cornea. 2017;36 (9):1155-1157. doi:10.1097/ICO.0000000000001254

\section{Publish your work in this journal}

Clinical Ophthalmology is an international, peer-reviewed journal covering all subspecialties within ophthalmology. Key topics include: Optometry; Visual science; Pharmacology and drug therapy in eye diseases; Basic Sciences; Primary and Secondary eye care; Patient Safety and Quality of Care Improvements. This journal is indexed on PubMed

Submit your manuscript here: https://www.dovepress.com/clinical-ophthalmology-journal
Central and CAS, and is the official journal of The Society of Clinical Ophthalmology (SCO). The manuscript management system is completely online and includes a very quick and fair peer-review system, which is all easy to use. Visit http://www.dovepress.com/ testimonials.php to read real quotes from published authors. 